Wartezeiten

\section{Beschäftigen Sie Ihre Patienten!}

Jeder Mensch fühlt sich ernst genommen und aufgewertet, wenn seine Zeit als wertvoll angesehen wird. Das gilt auch für den Arzt und noch mehr für seine Patienten. Mit den folgenden Tipps geben Sie Ihren Patienten das Gefühl, dass Sie mit ihrer Zeit sorgsam umgehen.

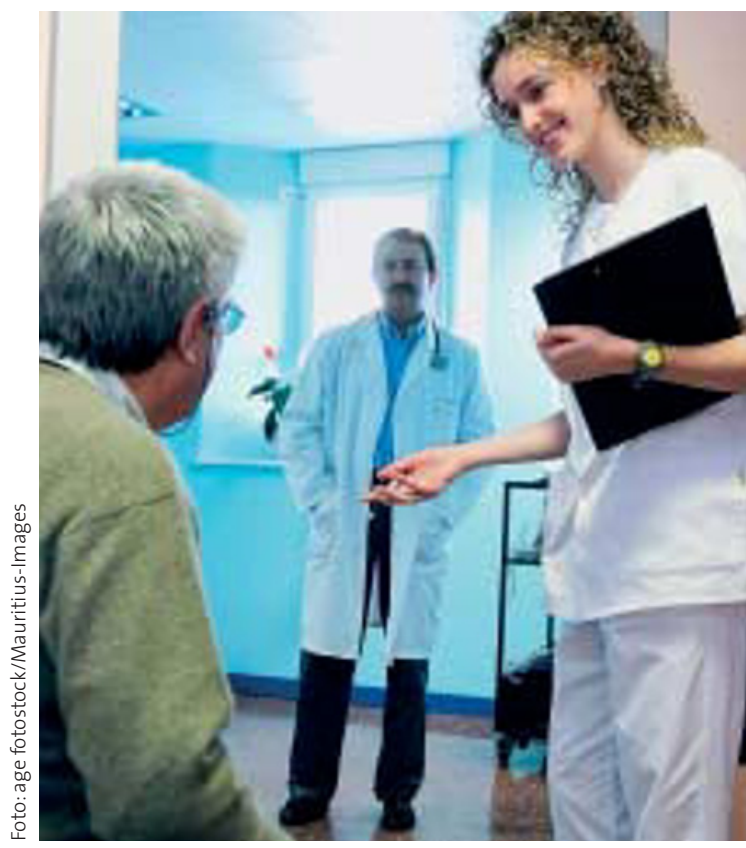

Sobald Ihr Patient die Praxis betritt, sollten Sie ihm das Gefühl geben: Wir kümmern uns um Sie!

- Beginnen Sie mit einer Aktion, sofort wenn der Patient hereinkommt! Es ist eine angenehme Überraschung für den Patienten, wenn umgehend etwas für ihn und mit ihm passiert, sobald er Ihre Praxis betritt. Das kann die Bitte um Abgabe einer Urinprobe sein, ein EKG oder eine Lungenfunktion, es kann auch eine delegierbare Behandlung durchgeführt werden, etwa eine Infusion oder Elektrotherapie. Subjektiv reduzieren solche Maßnahmen die Wartezeit auf null, denn es geschieht ja etwas.

Heißt es dagegen beim Hereinkommen „Nehmen Sie im Wartezimmer Platz", steigt bei vielen Patienten der Puls in der 9. Minute steil an, um in der 11. bis 13. Minute in eine massive Tachykardie überzugehen.
Der - meist unbewusste - Wunsch des Patienten ist es zunächst, wahrgenommen zu werden. Wird nach der Begrüßung sofort ein EKG geschrieben, dann wartet der Patient geduldig auf die Auswertung des Arztes und nimmt die Wartezeit bis zum eigentlichen Gespräch gerne in Kauf. Wichtig: Je freundlicher und zuvorkommender die Helferinnen bei ihren vorbereidesto angenehmer wird die Wartezeit für den Patienten.

\section{Kündigen Sie bestimmte Leistungen im Voraus an!} Noch geschickter gehen Sie vor, wenn Sie dem Patienten bestimmte technische Untersuchungen bereits bei der Terminvergabe ankündigen. Sie können sich das zur Gewohnheit machen. Denn die meisten Untersuchungen, gerade bei Patienten mit chronischen Leiden, etwa einer chronisch obstruktiven Lungenerkrankung, sind vorhersehbar. So können Sie schon im Voraus die Lungenfunkiton oder die Blutgasanalyse anordnen und vermeiden dadurch Wartezeiten, da Sie Arzt- und Helferinnenaktion in passender Weise verknüpfen.

\section{Kombinieren Sie langwierige und kurz dauernde Leistungen!}

Besonders zeitsparend - für Sie wie für den Patienten - ist es, wenn Sie bestimmte Aufgaben in die Zeitspanne legen, in der der Patient mit einer länger dauernden Untersuchung oder Behandtenden Tätigkeiten sind, lung beschäftigt ist. Haben Sie beispielsweise eine längere Infusion angeordnet, dann können Sie während dieser Zeit mit dem Patienten sprechen. Der Vorteil: Ihnen steht ein großes Zeitfenster zur Verfügung, Sie sind dementsprechend flexibel; der Patient wiederum wartet subjektiv überhaupt nicht auf Sie und kann die Praxis schneller verlassen, als wenn die Etappen nacheinander stattgefunden hätten.

\section{Helferinnenleistungen sind eine besondere Auszeichnung!}

Wenn Sie reine Helferinnenleistungen durchführen lassen, dann müssen Sie dies dem Patienten so vermitteln, dass er es als Kompliment für seine Selbstständigkeit und seine eigene Therapiekompetenz auffasst. „Sie kennen sich schon so gut aus, wir führen diese drei Infusionen durch, ohne dass Sie extra auf mich warten müssen; wir unterhalten uns dann beim vierten Termin."

Voraussetzung für eine solche Strategie sind genügend Behandlungsplätze und besonders kompetente Helferinnen. Diese müssen ihre Leistung mit Namenszeichen dokumentieren. Als Verantwortlicher müssen Sie ohne aktives Nachfragen erfahren, dass die Leistung durchgeführt wurde oder auch dass der Patient nicht zum Termin erschienen ist.

Dr. Roman Machens, ALLGEMEINARZT AUS LANDSHUT .

\section{Praxistipp}

5 Regeln, damit Ihre Patienten nicht unnötig lange warten!

- Aktion, sobald der Patient in die Praxis kommt

- Arzt- und Helferinnenleistungen beim selben Termin

- Leistungen im Voraus planen und dem Patienten ankündigen

- Langwierige und kurz dauernde Leistungen kombinieren

- Helferinnenleistungen als Vorzugsbehandlung vermitteln 